\title{
Editorial
}

\section{Semantic Web surveys and applications}

\author{
Pascal Hitzler ${ }^{\mathrm{a}}$ and Krzysztof Janowicz ${ }^{\mathrm{b}}$ \\ ${ }^{a}$ Kno.e.sis Center, Wright State University, USA \\ ${ }^{\mathrm{b}}$ University of California, Santa Barbara, USA
}

The primary purpose of a scientific journal is to be a service to the research community. It is up to the editors-in-chief and the editorial board to assess the needs of the community, and to tailor the journal in such a way that it addresses these needs as closely as possible.

It is for this reason that the Semantic Web journal publishes more than original research papers. In the last editorial [2], we discussed why it is fruitful for the Semantic Web community to have a forum for publications on tools and systems with high impact, and the last issue of the journal contained a collection of reports on some of the finest and most well-known Semantic Web tools currently available.

The present issue contains three surveys and one application paper. The surveys are responses to a special call which we issued in fall 2010, asking for abstracts first. Out of 37 submitted abstracts, we selected those which were most promising in addressing current and future needs of the Semantic Web community. It was only 6 for which we strongly encouraged the authors to write a full survey. Three of the resulting surveys are presented in this issue.

The quality we expect from survey articles is indeed very high. ${ }^{1}$ We expect them to be well-written and structured, comprehensive, and with a balanced presentation and coverage. They must be accessible to readers who are not dedicated specialists on the survey's topic. The covered material must be of timely importance to the broader Semantic Web community. Finally, a survey must provide a classification of the presented work that supports the reader in understand-

\footnotetext{
${ }^{1}$ See the explicit review criteria listed on the Semantic Web journal website, http://www.semantic-web-journal.net/.
}

ing the commonalities and differences between the reviewed approaches. In other words, surveys for the Semantic Web journal must have the potential to become well-known introductory and overview articles. Given these selection criteria, it is no surprise that one of the surveys in this issue is the currently most accessed paper on the Semantic Web journal website.

We consider high-quality surveys to be of major importance to the Semantic Web in its current state of development. The community is growing, there is significant uptake in the industry, but accessing the state of the art and staying up-to-date in the different subcommunities is a difficult and time-consuming process. While introductory textbooks can now be found on the market, ${ }^{2}$ these can only cover the basics and capture the knowledge and methods which are already stable over a certain period of time. The state of the art of many relevant current topics, however, is a constantly moving target, and books are not the right tool to quickly capture and deliver a snapshot of ongoing research. High-quality survey articles address this gap and thereby offer added value for the community. Thus, besides other paper types, the Semantic Web journal provides a forum for survey articles as well.

The last article in this issue is an application report yet another type of submission supported by the journal. Application reports in the Semantic Web journal describe deployed applications. The impact of these applications is a key review criterion, i.e., project use case applications or studies introduced in $\mathrm{Ph}$.D. theses or technical reports usually do not qualify due to lack of archival value and long-term impact. With the Semantic Web gaining momentum beyond research, re- 
ports on real applications with lasting impact are of major importance as bridgeheads for further application developments. At the same time, it appears to be rather difficult to convince industrial application developers to invest the time to write a report on their findings. By providing a prominent forum for such papers, however, the Semantic Web journals makes an attempt to stimulate such contributions. And indeed we would like to thank the authors of the paper, of Ontotext AD, for their contribution.

\section{References}

[1] D. Allemang and J. Hendler, Semantic Web for the Working Ontologist, Morgan Kaufmann, 2008.

[2] P. Hitzler and K. Janowicz, Semantic Web tools and systems, Semantic Web 2(1) (2011), 1-2.

[3] P. Hitzler, M. Krötzsch, and S. Rudolph, Foundations of Semantic Web Technologies, Chapman \& Hall/CRC, 2009. 